\title{
Improving the osteogenesis of human bone marrow mesenchymal stem cell sheets by microRNA-2I- loaded chitosan/hyaluronic acid nanoparticles via reverse transfection
}

\author{
This article was published in the following Dove Press journal: \\ International Journal of Nanomedicine \\ 17 May 2016 \\ Number of times this article has been viewed
}

\section{Zhongshan Wang,' \\ Guangsheng $\mathrm{Wu},{ }^{2,3}$ \\ Mengying Wei, ${ }^{4}$ Qian Liu, Jian Zhou,' Tian Qin,' Xiaoke Feng,' Huan Liu,' Zhihong Feng,' Yimin Zhao'}

'State Key Laboratory of Military Stomatology \& National Clinical Research Center for Oral Diseases \& Shaanxi Key Laboratory of Oral Diseases, Department of Prosthodontics, ${ }^{2}$ State Key Laboratory of Military Stomatology \& National Clinical Research Center for Oral Diseases \& Shaanxi Engineering Research Center for Dental Materials and Advanced Manufacture, Department of Periodontology, School of Stomatology, The Fourth Military Medical University, Xi'an, ${ }^{3}$ Qingdao First Sanatorium, Jinan Military Region, Qingdao, Shandong Province, ${ }^{4}$ Department of Biochemistry and Molecular Biology, The Fourth Military Medical University, Xi'an, People's Republic of China
Correspondence: Zhihong Feng; Yimin Zhao

State Key Laboratory of Military Stomatology \& National Clinical Research Center for Oral Diseases \& Shaanxi Key Laboratory of Oral Diseases, Department of Periodontology, I 45 West Changle Road, Xi'an 7I0032, People's Republic of China

Tel/fax +86 2984776465

Email 398823267@qq.com; zhaoymdentist@I63.com

\begin{abstract}
Cell sheet engineering has emerged as a novel approach to effectively deliver seeding cells for tissue regeneration, and developing human bone marrow mesenchymal stem cell (hBMMSC) sheets with high osteogenic ability is a constant requirement from clinics for faster and higher-quality bone formation. In this work, we fabricated biocompatible and safe chitosan (CS)/hyaluronic acid (HA) nanoparticles (NPs) to deliver microRNA-21 (miR-21), which has been proved to accelerate osteogenesis in hBMMSCs; then, the CS/HA/miR-21 NPs were cross-linked onto the surfaces of culture plates with $0.2 \%$ gel solution to fabricate miR-21-functionalized culture plates for reverse transfection. hBMMSC sheets were induced continuously for 14 days using a vitamin C-rich method on the miR-21-functionalized culture plates. For the characterization of CS/HA/miR-21 NPs, the particle size, zeta potential, surface morphology, and gel retardation were sequentially investigated. Then, the biological effects of hBMMSC sheets on the miR-21-functionalized culture plates were evaluated. The assay results demonstrated that the hBMMSC sheets could be successfully induced via the novel reverse transfection approach, and miR-21 delivery significantly enhanced the in vitro osteogenic differentiation of hBMMSC sheets in terms of upregulating calcification-related gene expression and enhancing alkaline phosphatase production, collagen secretion, and mineralized nodule formation. The enhanced osteogenic activity of hBMMSC sheets might promisingly lead to more rapid and more robust bone regeneration for clinical use.
\end{abstract}

Keywords: human bone marrow MSCs, cell sheets, microRNA, chitosan, nanoparticles, osteogenic differentiation

\section{Introduction}

Recently, stem-cell therapy has become a nascent but fast-growing area in the field of tissue regeneration. ${ }^{1}$ Stem-cell therapy has proved effective clinically and could potentially lead to robust treatment outcomes for predictable tissue regeneration. . $^{2,3}$ However, the traditional transplanted models, such as direct cell suspension injection or combination with biomaterials, have tended to present low efficacy, as well as poor survival of the seeding cells, which might impede the expected therapeutic effects in humans. ${ }^{4}$ At the same time, cell sheet engineering has emerged as a novel approach to effectively deliver seeding cells with good preservation of in vitrosecreted extracellular matrix (ECM) around stem cells. ${ }^{5}$ Cell sheets were recently induced using a vitamin $\mathrm{C}(\mathrm{Vc})$ inducing method because of increased cell matrix production and deposition. ${ }^{6}$ This cell processing approach could effectively preserve 
intact cell-cell junctions comprising various biological and mechanical properties and could increase the cell survival rate and reduce cell loss during cell sheet transplantation. ${ }^{6,7}$ The cell sheets could be applied layer by layer and could attach well to defective areas of tissues, allowing for either direct in vivo transplantation or combination with biomaterials in tissue engineering. ${ }^{8}$ This technique has proved effective in treating esophageal ulcerations, ${ }^{9}$ periodontal tissue destruction, ${ }^{3}$ corneal dysfunction, ${ }^{10}$ myocardial infarction, ${ }^{2}$ and so on. Thus, we expect that producing higher-quality cell sheets with in vitro-induced specific lineage differentiation capacities would result in superior biological performance for specific tissue regeneration.

Over the last decade, the delivery of various therapeutic agents, such as DNA, small interfering RNAs, and microRNAs (miRNAs, miR), into stem cells has been a fundamental technology for studying the biological properties of target cells, as well as for inducing improved clinical therapeutic results. ${ }^{11}$ Among these agents, with a length of only 20-30 nucleotides, miRNAs have emerged as critical regulators of endogenous genes, binding primarily to the $3^{\prime}$ untranslated region of target messenger RNAs controlling the level of messenger RNA at the posttranscriptional level. ${ }^{11,12}$ As such, miRNAs provide a potentially less harmful strategy for controlling the specific differentiations of stem cells in vitro and in vivo. ${ }^{13}$ Although specific miRNAs have been widely delivered into isolated stem cells, they have only rarely been delivered into cell sheets, especially with an efficient reverse transfection approach. ${ }^{8}$ It would be of great significance to explore efficient and practical miRNA delivery approaches during cell sheet formation for enhanced clinical performance.

Vectors are normally required for the protection and efficient intracellular delivery of miRNAs. ${ }^{14}$ Nonviral gene delivery systems, such as chitosan (CS)/hyaluronic acid (HA) nanoparticles (NPs), have been proposed in terms of safety concerns, as well as for their excellent biocompatibility and biodegradability and their minimal host immune response compared to viral vectors. ${ }^{15-17}$ The positive charges of CS contribute to electrostatic interactions with negatively charged miRNAs for the delivery of transfection agents, which are also responsible for their capacity to interact with negatively charged cell surfaces. ${ }^{18} \mathrm{HA}$ is an anionic polysaccharide naturally found in humans, which has been widely used for bioabsorbable scaffolds and drug delivery carriers. ${ }^{19}$ In this study, the inclusion of an anionic polymer (HA) seemed to increase the stability of CS particles in plasma and to induce an even size distribution of NPs. ${ }^{19-21}$ In addition,
HA has the ability to bind to various cellular receptors, such as CD44, which is expressed in normal human bone marrow mesenchymal stem cells (hBMMSCs), cancerous cells, and so on. ${ }^{21,22}$ This property would increase the transfection efficacy of target cells and would reduce side effects. ${ }^{22}$

In the present design, HA was associated with CS through polyelectrolyte complexion to form CS/HA NPs, which were combined with miR-21s through electrostatic interactions to form miR-21-loaded CS/HA NPs. Then, the $\mathrm{CS} / \mathrm{HA} / \mathrm{miR}-21 \mathrm{NPs}$ were coated on to culture plates through cross-linking with $0.2 \%$ gel solution, ${ }^{15,17}$ after which the isolated hBMMSCs were seeded on to the functionalized culture plates and were induced into cell sheets based on a Vc-inducing method. ${ }^{6}$ Further, we evaluated the transgene expression efficacy and osteogenesis-enhancing effects on hBMMSC sheets. We believe that this novel coating could contribute to enhanced hBMMSC sheets with rapid and excellent osteogenic capabilities for clinical use.

\section{Materials and methods Materials}

CS was obtained from Jinke Co. Ltd (Zhejiang, People's Republic of China), with a molecular weight of $100 \mathrm{kDa}$ and a deacetylation degree of $90 \%$. HA, with a molecular weight of $10 \mathrm{kDa}$, was purchased from CP Freda Pharmaceutical Co., Ltd (Shangdong, People's Republic of China). Tripolyphosphate (TPP) was supplied by Sigma-Aldrich Co. (St Louis, MO, USA). The hsa-miR-21 mimics (5'-uagcuuaucagacu gauguugadTdT-3'; 5'-ucaacaucagucugauaagcuadTdT-3') were purchased from GenePharma (Shanghai, People's Republic of China). Gelatin powder (type A) and vitamin C were purchased from Sigma-Aldrich Co. $\alpha$-minimum essential medium ( $\alpha$-MEM) and fetal bovine serum were obtained from Thermo Fisher Scientific (Waltham, MA, USA). Fluorescently labeled monoclonal antibodies, including STRO-1, were acquired from BD Biosciences (San Jose, CA, USA); CD146, CD29, CD34, and CD45 were purchased from Biolegend (San Diego, CA, USA). Primers for real-time polymerase chain reaction (PCR) were purchased from Sangon Biotechnology Co. (Shanghai, People's Republic of China). Antibodies against collagen type I $\alpha 1$ (COL-I), runt-related transcription factor 2 (RUNX2), osteopontin (OPN), and osteocalcin (OCN) were all provided by Santa Cruz Biotechnology Inc. (Dallas, TX, USA).

\section{CS/HA/miR-2I NP preparation}

$\mathrm{CS} / \mathrm{HA}$ NPs were produced using the ionotropic gelation technique with different CS:TPP:HA volume ratios, 
as described in our previous study. ${ }^{16}$ An optimal mixture with a CS:TPP:HA volume ratio of 1:0.15:0.1 was adopted to prepare NPs. ${ }^{16} \mathrm{NPs}$ were formed instantaneously upon the dropwise addition of TPP/HA solution to a fixed volume of CS solution under magnetic stirring for 10 minutes. CS/HA/ miR-21 NPs were prepared by adding the required volume of $50 \mu \mathrm{M}$ miR-21s to the CS/HA NP systems by gentle pipetting to form complexes of a selected N/P ratio, which was defined as the molar ratio of the positive CS amino group to the negative miRNA phosphate group. Subsequently, the mixture was vortexed rapidly for 3-5 seconds and was left for 1 hour at room temperature for the complexes to form completely.

\section{Characterization of CS/HA/miR-2I NPs}

Particle size, zeta potential, surface morphology, and gel retardation analysis

The surface charge and diameter of CS/HA NPs were determined by dynamic light scattering using a Zetasizer Nano ZS instrument (Malvern Instruments, Malvern, UK) at $25^{\circ} \mathrm{C}$. For morphology observation, samples were dried at room temperature on copper grids and then photographed using a transmission electron microscope (TEM; FEI Company, Tecnai G2, USA). For gel retardation analysis, CS/HA NPs and naked miR-21s at various N/P ratios (1:1, 5:1, 10:1, 15:1, and $20: 1)$ were loaded on to a $2 \%$ agarose gel containing ethidium bromide in Tris-borate ethylenediaminetetraacetic acid buffer at $\mathrm{pH}$ 8.0. The samples were run on the gel at $120 \mathrm{~V}$ for 20 minutes and then photographed using a GDS-8000 image-acquisition system (UVP, USA).

\section{Preparation of $\mathrm{CS} / \mathrm{HA} / \mathrm{miR}-2 \mathrm{I}$ NP-coated culture plates and surface characterization}

The miR-21 coating was achieved by cross-linking the $\mathrm{CS} / \mathrm{HA} / \mathrm{miR}-21 \mathrm{NPs}$ with $0.2 \%$ gelatin solution on culture plates. ${ }^{15}$ In brief, $0.2 \%$ gelatin (w/v) was dissolved in distilled water by heating and gentle swirling in a $60^{\circ} \mathrm{C}$ water bath for 15 minutes; then, it was sterilized by high-pressure steam and was cooled slowly at room temperature and stored at $4^{\circ} \mathrm{C}$. Subsequently, an appropriate amount of CS/HA NP solution was mixed with $50 \mu \mathrm{M}$ miR-21 solution to produce $\mathrm{CS} / \mathrm{HA} /$ miR-21 NP solution (N/P =20:1); then, different volumes of $\mathrm{CS} / \mathrm{HA} / \mathrm{miR}-21 \mathrm{NP}$ solutions containing $150 \mathrm{pmol}, 300 \mathrm{pmol}$, and 450 pmol miR-21 were mixed evenly with an equal volume of $60 \mu \mathrm{L}$ of $0.2 \%$ gelatin. The mixtures were pipetted on to six-well culture plates and were dispersed using $200 \mu \mathrm{L}$ tips as evenly as possible, and they were then air dried in a laminar flow cabinet and stored at $4^{\circ} \mathrm{C}$.
For surface characterization, the prepared miR-21-coated culture plates were fixed in $2.5 \%$ glutaraldehyde, dehydrated in a graded ethanol series, and freeze dried. After sputter coating with gold, the surface morphology was observed with the field emission scanning electron microscope (FE-SEM) (Hitachi S-4800; EIKO, Shawnee, KS, USA).

To determine whether miR-21s were stably retained within CS/HA NPs after CS/HA/miR-21 NPs were coated on to culture plates, Cy3-labeled miR-21 and fluorescein isothiocyanate (FITC)-labeled CS were adopted to prepare fluorescencelabeled CS/HA/miR-21 NPs, and then, the same NP coatings were fabricated as earlier. The $\mathrm{Cy} 3$ and FITC fluorescence signals were observed under fluorescence microscopy (Olympus Corporation, Tokyo, Japan) to evaluate the distribution and locations of miR-21s and CS/HA NPs, respectively.

Contact angles were measured by the sessile drop method on an Easy Drop Standard instrument (KRUSS GmbH, Hamburg, Germany) at room temperature. The drop shapes of distilled water on different surfaces were photographed, and the contact angles and free energy were calculated using DSA1 software (KRUSS GmbH).

\section{Cell culture and characterization of stemness}

Primary hBMMSCs were isolated according to a previous protocol. ${ }^{15}$ Briefly, fresh cancellous bone fragments were obtained from orthognathic patients (22-26 years of age, $\mathrm{n}=3$ ) via mandibular angle resection, with written informed consent and Institutional Review Board approval from the Stomatological Hospital of the Fourth Military Medical University. For cell isolation, a syringe device was used to pour $\alpha$-MEM against the fragments gently and repeatedly until the fragments displayed a white appearance. Then, the mixed cell types were collected and seeded on a six-well plate (Corning Incorporated, Corning, NY, USA) containing $\alpha$-MEM supplemented with $10 \%$ fetal bovine serum (Thermo Fisher Scientific), $0.292 \mathrm{mg} / \mathrm{mL}$ glutamine (Sigma-Aldrich Co.), $100 \mathrm{U} / \mathrm{mL}$ penicillin and $100 \mathrm{mg} / \mathrm{mL}$ streptomycin (both from Sigma-Aldrich Co.) in a humidified atmosphere at $37^{\circ} \mathrm{C}$ with $5 \% \mathrm{CO}_{2}$; the culture media were refreshed every 2 days. hBMMSCs from passages 2-5 were used in the subsequent experiments.

Cell surface markers of hBMMSCs (P3) were determined by flow cytometry analysis using a previous method. ${ }^{23}$ Briefly, $2 \times 10^{5}$ cells were trypsin collected and transferred to each microtube, after which antibodies, such as STRO-1, CD146, CD29, CD34, and CD45, were added to each microtube, based on the manufacturer's instructions; 
cell suspensions without any added antibody served as controls. All the microtubes were incubated at $37^{\circ} \mathrm{C}$ in the dark for 40 minutes and then washed with phosphate-buffered saline (PBS) three times. Finally, the cells were resuspended in $300 \mu \mathrm{L}$ of PBS for the analysis of cell surface markers using a flow cytometry cell sorting Vantage Cell Sorter (Becton \& Dickinson, Mountain View, USA). The data were analyzed using the Mod-Fit 2.0 cell cycle analysis program (Becton \& Dickinson).

To assess the ability of osteogenic/adipogenic differentiation, the cells (P3) were seeded on to six-well dishes at a concentration of $2 \times 10^{5}$ cells/well and cultured in complete medium until they reached $80 \%$ confluence. To assess osteogenic ability, the osteoinductive medium (complete medium supplemented with $50 \mu \mathrm{g} / \mathrm{mL}$ ascorbic acid, $10 \mathrm{nM}$ dexamethasone, and $10 \mathrm{mM} \beta$-glycerophosphate) was switched and refreshed every 3 days. To assess adipogenic ability, the adipogenic medium (complete medium supplemented with $100 \mathrm{nM}$ dexamethasone, $0.5 \mathrm{mM}$ 3-isobutyl1-methylxanthine, and $50 \mathrm{mM}$ indomethacin) was also changed and refreshed at 3-day intervals. After 4-week osteogenic induction or 2 -week adipogenic induction, the cells were fixed in 4\% paraformaldehyde and stained with alizarin red or fresh Oil Red $\mathrm{O}$ solution, respectively. The mineralization nodules and lipid droplets were observed photographically (IX70; Olympus Corporation).

\section{Cytotoxicity and cell transfection}

For cytotoxicity determination, the lactate dehydrogenase (LDH) activity was used as an index of cytotoxicity in the culture medium. ${ }^{15}$ Briefly, after 24 hours of transfection at different miR-21 concentrations (150 pmol/well, 300 pmol/ well, and $450 \mathrm{pmol} /$ well), the culture medium was collected and centrifuged, and the supernatant was used for the $\mathrm{LDH}$ activity assay. The LDH activity was determined spectrophotometrically, according to the manufacturer's instructions. Three parallel experiments in each group were conducted.

To assess the transfection efficacy of CS/HA/miR-21 NPs on culture plates, Cy3-labeled miRNAs and FITClabeled CS were used to prepare fluorescence-labeled CS/HA/miR-21 NPs. The cells (P3) were seeded on to six-well miR-21-functionalized culture plates at a density of $2 \times 10^{5}$ cells/well. At the time points of 24 hours and 14 days, the cells were collected in trypsin, washed twice with PBS, and fixed in 1\% paraformaldehyde in PBS. The Cy3 fluorescence emitted by the cells after internalization was measured with a flow cytometer (FACScalibur; BD Biosciences). As a negative control, untreated cells were subjected to the same process. Three biological replicates of each sample were analyzed.

To track the fluorescence of internalized NPs, fluorescently labeled CS/HA/miR-21 NPs were used, and the cells were transfected at a concentration of $300 \mathrm{pmol} \mathrm{miR-21/well.}$ At the time points of $\mathrm{d} 1, \mathrm{~d} 3, \mathrm{~d} 5, \mathrm{~d} 7, \mathrm{~d} 10$, and $\mathrm{d} 14$, the cells were immediately fixed and washed in PBS twice. Then, the nuclei of the cells were stained with 4',6-diamidino-2phenylindole (DAPI; Sigma-Aldrich Co.). Subsequently, the FITC, Cy3, and DAPI fluorescence signals were observed via fluorescence microscopy (Olympus Corporation) to evaluate the distribution of the $\mathrm{CS} / \mathrm{HA} / \mathrm{miR}-21 \mathrm{NPs}$ at each time point.

\section{In vitro osteogenesis of the hBMMSC sheets}

To assess the in vitro osteogenesis of the miR-21-delivered hBMMSC sheets, the control group without delivery (noncoated control) and the control group with naked CS/HA NP coating (CS/HA control) were used. After 14 days of cell sheet induction, the hBMMSC sheets were further incubated with the osteoinductive medium (complete medium supplemented with $50 \mu \mathrm{g} / \mathrm{mL}$ ascorbic acid, $10 \mathrm{nM}$ dexamethasone, and $10 \mathrm{mM} \beta$-glycerophosphate) for 14 days. The osteogenesis-related gene expression, alkaline phosphatase (ALP) production, and collagen secretion, as well as ECM mineralization, were systemically assessed.

Osteogenesis-related gene expression was evaluated using real-time PCR. Briefly, total RNA was extracted by using TRIzol reagent (Thermo Fisher Scientific), and complementary DNA synthesis (Takara Bio, Otsu, Japan) was then conducted. The expression levels of osteogenesisrelated genes, including $C O L-I, R U N X 2, O P N$, and $O C N$, were quantified using a CFX Connect ${ }^{\mathrm{TM}}$ Real-Time PCR Detection System (Bio-Rad Laboratories Inc., Hercules, CA, USA) with SYBR Green Master (Hoffman-La Roche Ltd, Basel, Switzerland). The relative expression levels of target genes were normalized to the expression of the housekeeping gene glucuronidase, beta (GUSB). The forward/reverse primers for the selected genes are listed in Table 1.

For Western blot analysis, cell sheet samples were lysed in lysis buffer (Sigma-Aldrich Co.) supplemented with $1 \mathrm{mM}$ phenylmethanesulfonyl fluoride (PMSF) (Hoffman-La Roche). Then, the total protein concentrations were measured using a bicinchoninic acid protein assay kit (Beyotime, Shanghai, People's Republic of China). Next, $40 \mu \mathrm{g}$ of cell lysates were added to each lane of $10 \%$ sodium dodocyl 
Table I Target cDNA primer sequences used for real-time PCR

COLI

Forward: 5'-GAGGGCCAAGACGAAGACATC-3'

Reverse: 5'-CAGATCACGTCATCGCACAAC-3'

RUNX2

Forward: 5'-TGGTTACTGTCATGGCGGGTA-3'

Reverse: 5'-TCTCAGATCGTTGAACCTTGCTA-3' OPN

Forward: 5'-CAGTTGTCCCCACAGTAGACAC-3'

Reverse: 5'-GTGATGTCCTCGTCTGTAGCATC-3' OCN

Forward: 5'-CCCAGGCGCTACCTGTATCAA-3'

Reverse: 5'-GGTCAGCCAACTCGTCACAGTC-3'

GUSB (housekeeping gene)

Forward: 5'-GTCTGCGGCATTTTGTCGG-3'

Reverse: 5'-CACACGATGGCATAGGAATGG-3'

Abbreviations: $c D N A$, complementary DNA; PCR, polymerase chain reaction; COLI, collagen type $|\alpha| ; R U N X 2$, runt-related transcription factor 2; OPN, osteopontin; OCN, osteocalcin; GUSB, glucuronidase, beta.

sulfate-polyacrylamide gel electrophoresis (SDS-PAGE) gels, separated based on molecular weight, and electrotransferred to polyvinylidene fluoride membranes (EMD Millipore, Billerica, MA, USA). After blocking with 5\% nonfat milk for 2 hours, the membranes were incubated in specific primary antibodies against COL-I, RUNX2, OPN, and OCN overnight at $4^{\circ} \mathrm{C}$. Subsequently, the membranes were incubated in a horseradish peroxidase-conjugated anti-rabbit or anti-mouse secondary antibody (CoWin Biotech Co., Ltd, Beijing, People's Republic of China) for 1 hour at room temperature. The membranes were developed using the Western Light Chemiluminescent Detection System (Peiqing, Shanghai, People's Republic of China). All the assays were repeated in triplicate.

To assess ALP production, at the predestinated time points, the cell sheets were fixed and incubated with $2 \mathrm{~mL}$ of staining fluid (a mixture of $10 \mathrm{~mL}$ of buffer, $33 \mu \mathrm{L}$ of 5-bromo-4-chloro-3-indolylphosphate, and $66 \mu \mathrm{L}$ of nitroblue tetrazolium chloride; Beyotime) for 30 minutes at room temperature in the dark, and they were then washed with PBS. ALP activity was expressed as the integrated optical density (IOD) of the blue staining of the cytoplasm on representative images. The IOD was analyzed using Image-Pro Plus 6.0 software (Media Cybernetics, Silver Spring, MD, USA).

To assess collagen secretion, the fixed cell sheets were immersed in $0.1 \mathrm{wt} \%$ Sirius red (Sigma-Aldrich Co.) with saturated picric acid for 1 hour, and then, the secreted collagen fibers were observed microscopically. Subsequently, the stain was dissolved with $1 \mathrm{~mL}$ of destain solution $(0.2 \mathrm{M}$
$\mathrm{NaOH} /$ methanol 1:1), and the absorbance at $540 \mathrm{~nm}$ was quantitatively measured.

For ECM mineralized nodule staining, the fixed cell sheets were stained with $1 \mathrm{wt} \%$ alizarin red for 3 minutes to display the mineralized nodules and were washed with PBS three times, dried at room temperature, and observed under a microscope. Finally, the mineralization nodules were dissolved in $10 \%$ cetylpyridinium chloride in $10 \mathrm{mM}$ sodium phosphate ( $\mathrm{pH} 7)$, and the OD values were measured at $620 \mathrm{~nm}$ using a spectrophotometer for statistical analysis.

\section{Statistical analysis}

The results were collected and analyzed with SPSS 17.0 software (SPSS Inc., Chicago, IL, USA), and all values are expressed as the mean \pm standard deviation. The level of significance was determined using two-way analysis of variance combined with SNK-q tests or Student's $t$-test. A $P$-value of $<0.05$ was considered statistically significant.

\section{Results \\ NP characterization}

Particle size, zeta potential, TEM observation, and gel retardation analysis

The CS/HA NPs were prepared by ionic gelation among positively charged CS and negatively charged TPP and HA. According to our previous studies, an optimal mixture with a CS:TPP:HA volume ratio of 1:0.15:0.1 was adopted to prepare NPs. The morphologies of the NPs were observed by TEM (Figure 1A and B), and the typical images illustrated that the NPs were finely dispersed in the visual field and displayed spherical shapes. As Figure 1C shows, the binding capacity of CS/HA NPs with miR-21 was evaluated by gel retardation assay. The $\mathrm{CS} / \mathrm{HA} / \mathrm{miR}-21 \mathrm{NPs}$ were previously prepared at various $\mathrm{N} / \mathrm{P}$ ratios $(1: 1,5: 1,10: 1$, 15:1, and 20:1). The results illustrated that the migrations of miR-21 in agarose gel were completely retarded when the $\mathrm{N} / \mathrm{P}$ ratio was 20:1, indicating the complete combination of miRNA-21s with CS/HA NPs. For the subsequent study, we applied CS/HA/miR-21 NPs with a CS:TPP:HA ratio of 1:0.15:0.1 and an N/P ratio of 20:1. The average diameter of the NPs was $181.3 \pm 12.55 \mathrm{~nm}$ (Figure 1D), and the average zeta potential was $35.2 \pm 4.37 \mathrm{mV}$ (Figure 1E).

\section{Fabrication and surface morphology of miR-2I-functionalized culture plates}

Scanning electron micrographs (Figure 2A) showed that the unmodified surface of the culture plates was relatively smooth; the gel-coated surface displayed highly ordered 

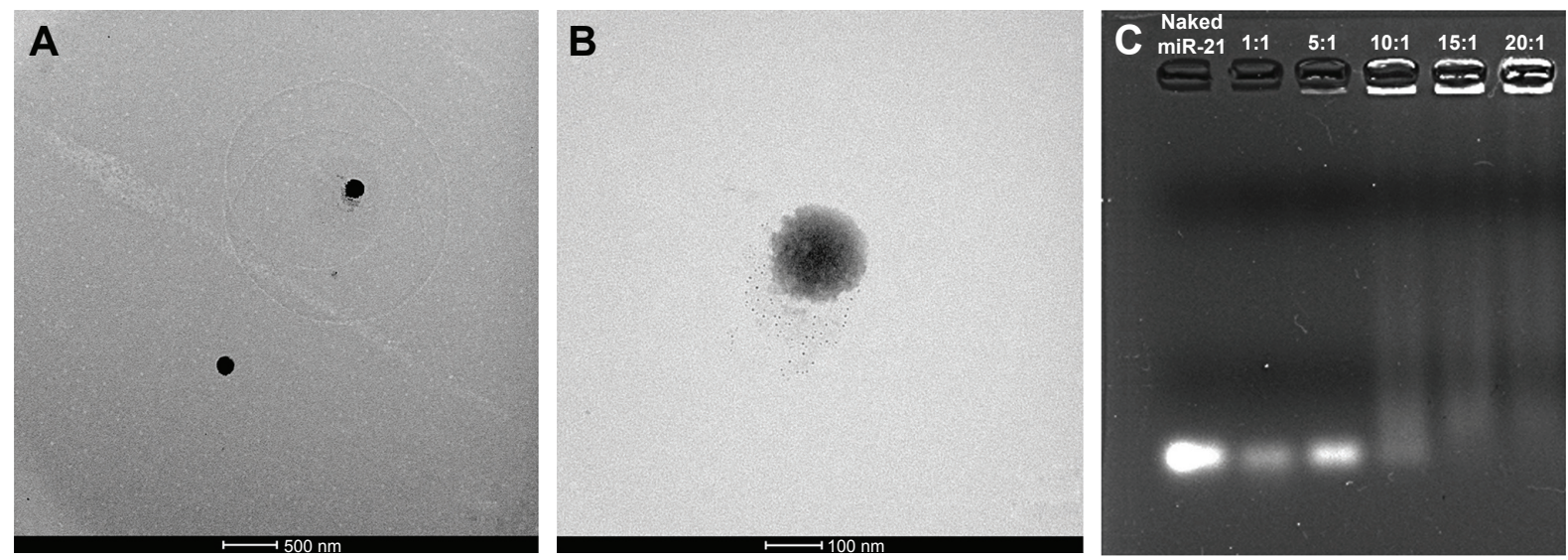

D

Size distribution by intensity

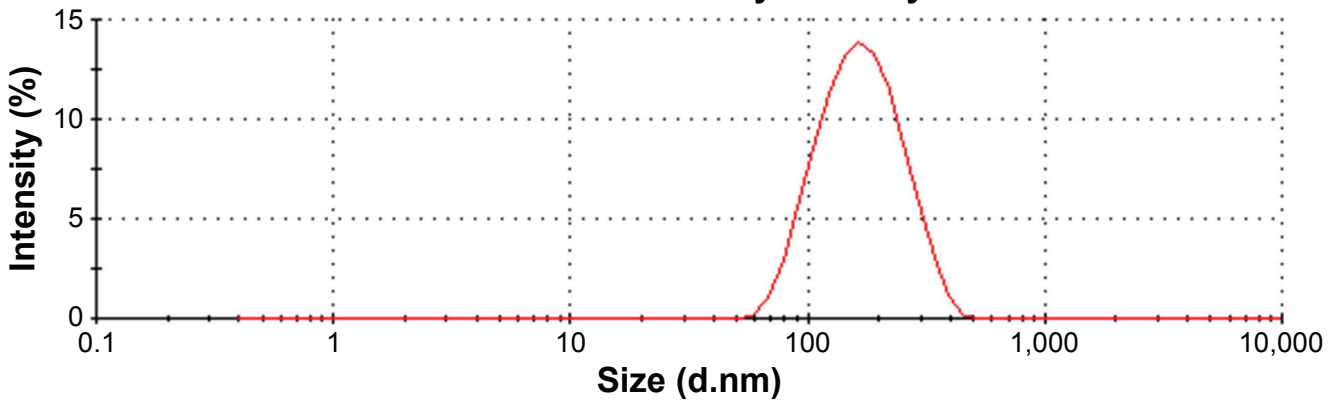

E

Zeta potential distribution

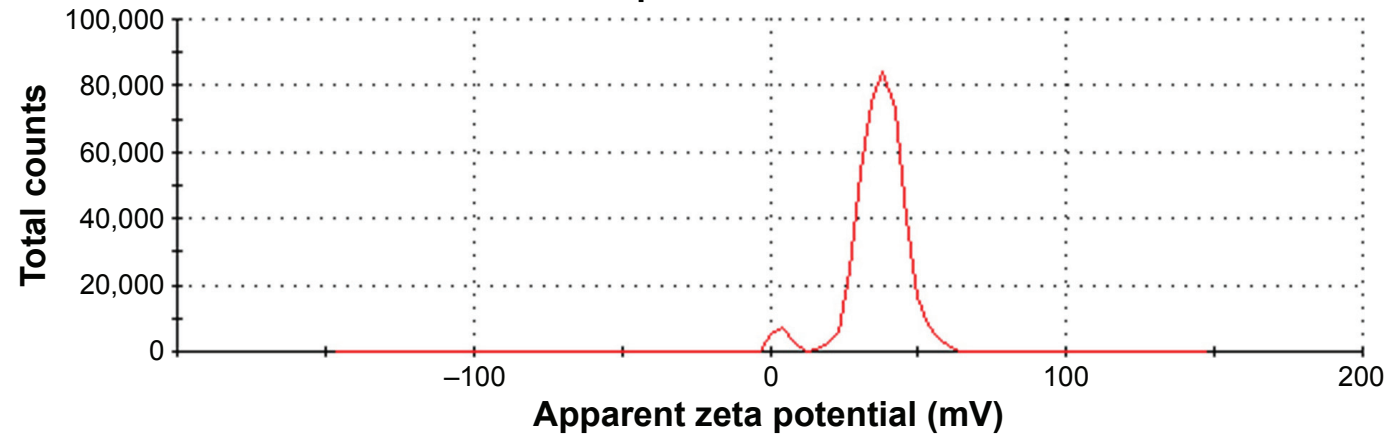

Figure I The characterizations of NPs.

Notes: (A and B) TEM images of CS/HA NPs with different magnifications (20,500x in A, and 105,000x in B). (C) Gel retardation analysis of CS/HA/miR-2I NPs; lane I, naked miR-2I; lanes 2-6, CS/HA/miR-2I NPs prepared at N/P ratios of I:I, 5:I, I0:I, I5:I, and 20:I. (D) The size distribution of the CS/HA/miR-2I NPs produced by an optimal mixture with a CS:TPP:HA volume ratio of I:0.15:0.1. (E) The zeta potential distribution of the CS/HA/miR-2I NPs.

Abbreviations: NPs, nanoparticles; TEM, transmission electron microscope; CS, chitosan; HA, hyaluronic acid; miR, microRNA; TPP, tripolyphosphate.

and aligned gel proteins; and the miR-21-functionalized surface presented uniform and sleek CS/HA/miR-21 NPs, with diameters ranging from $60 \mathrm{~nm}$ to $300 \mathrm{~nm}$, forming a monolayer and embedded tightly in the gel proteins.

Contact angle measurements showed that the gel-coated surface and miR-21-functionalized surface had increased surface energy. As Figure 2B indicates, the water contact angle of the unmodified surface was $\sim 83.2^{\circ}$, the gel-coated surface showed higher hydrophilia, with a contact angle of $47.5^{\circ}$, and the miR21-functionalized surface displayed similar hydrophilia, with a contact angle of $68.7^{\circ}$. The changes in the surface chemistry led to significantly different water contact angles.

Fluorescence microscopy was used to evaluate the distributions of FITC-labeled CS and Cy3-labeled miR-21s, and the images (Figure 2C) demonstrated that both the FITC-labeled CS and Cy3-labeled miR-21s were spread evenly on the substrates of the culture plates, and the merged image presented a high degree of overlap, indicating that the miR-21s were stably retained within the CS/HA NPs after the CS/HA/miR-21 NPs were coated on to culture plates. 


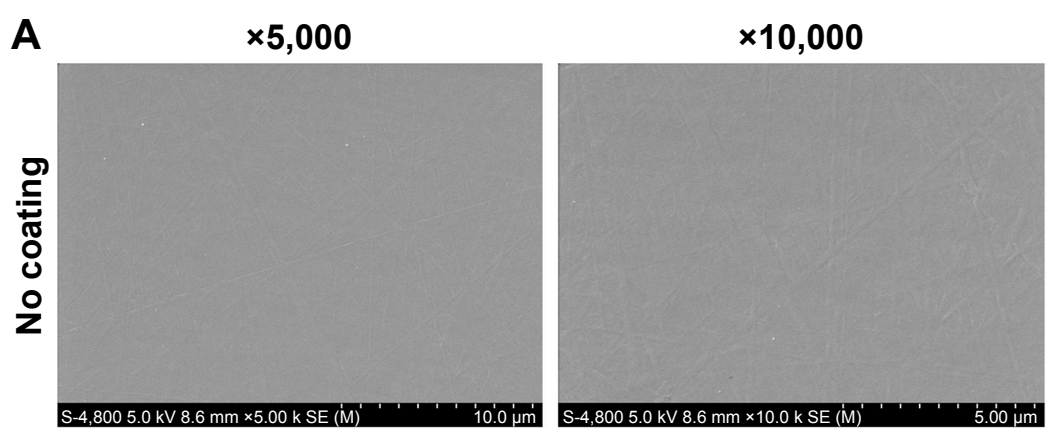

$\times 20,000$
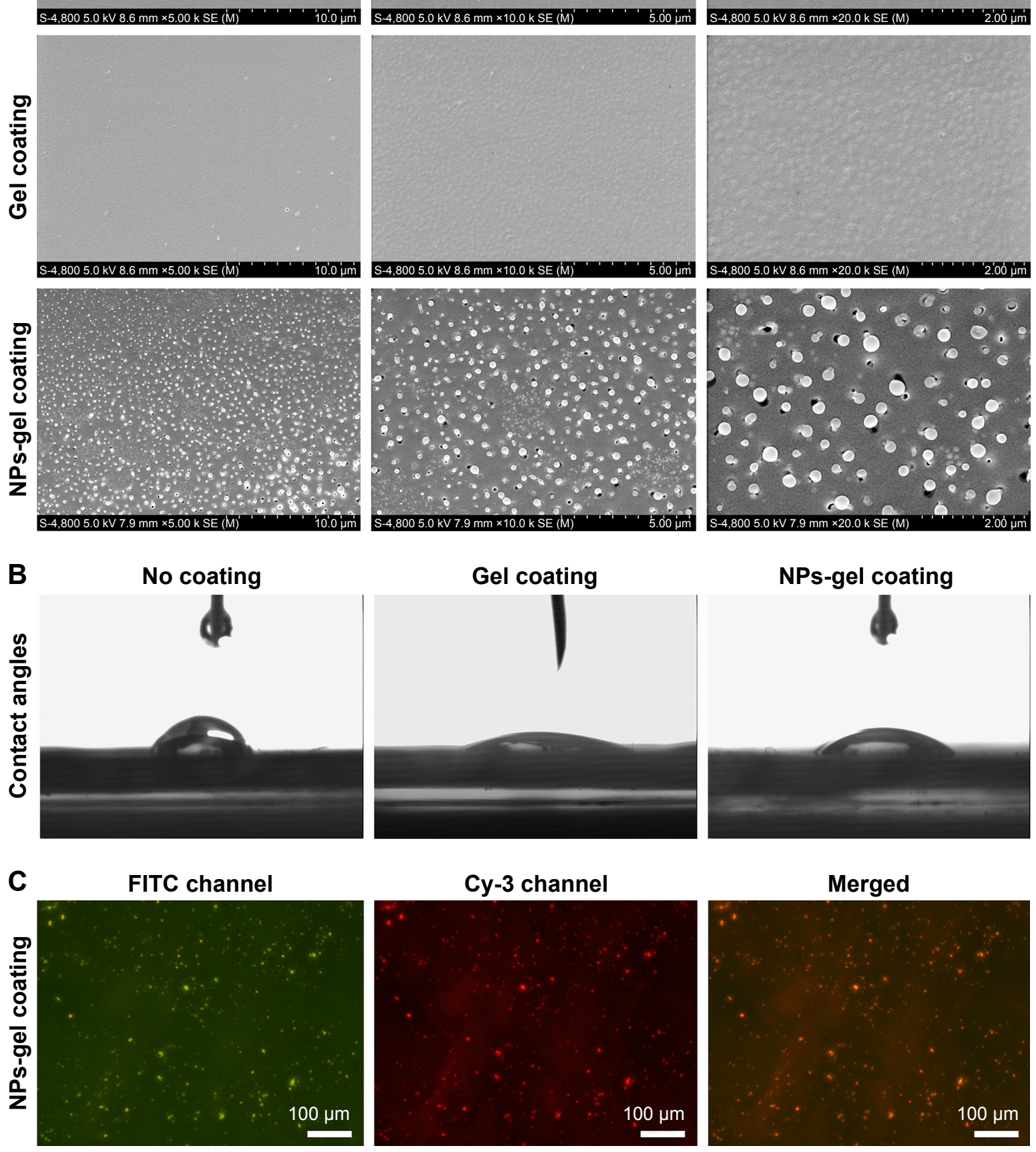

Figure 2 The characterizations of CS/HA/miR-2I NP-coated surfaces of culture plates.

Notes: (A) Surface morphologies of culture plates before and after CS/HA/miR-2I NP coating as inspected by SEM with increasing magnification. No coating: unmodified surface; gel coating: $0.2 \%$ gel solution-coated surface; NPs-gel coating: CS/HA/miR-2I NP-coated surface. (B) The measurement of water contact angles on different surfaces. (C) Fluorescence observation of CS/HA/miR-2I NP-coated surfaces (200x). The pictures illustrate FITC-labeled CS (green), Cy3-labeled miR-2Is (red), and the merged images. Abbreviations: CS, chitosan; HA, hyaluronic acid; miR, microRNA; NPs, nanoparticles; SEM, scanning electron microscope; FITC, fluorescein isothiocyanate. 


\section{Isolation and identification of hBMMSCs}

Primary hBMMSCs were successfully isolated and passaged. As Figure 3A shows, the primary cells presented a typical spindle-shaped morphology. One of the key properties of any MSC line is a capacity for multiple-direction differentiation. In terms of osteogenic differentiation capability, alizarin redpositive mineralized nodules could be observed following 4 weeks of incubation (Figure 3B), which provided strong evidence of the osteogenic potential of hBMMSCs. After 2 weeks of adipogenic induction, the microscopic oil red O-positive lipid droplets were widely formed in the cells (Figure 3C), indicating their adipogenic differentiation potential. The cell surface markers of hBMMSCs were determined by flow cytometry (Figure 3D), and the results revealed that the cells were positive for mesenchymal-associated markers, such as STRO-1, CD146, and CD29, while they were negative for hematopoietic markers, such as CD34 and CD45. These results indicated that hBMMSCs were successfully isolated and cultured.

\section{Cytotoxicity assay and transfection efficiency of the miR-2I-functionalized surface}

The cytotoxicity of different substrates was assessed by LDH activity in the culture media after 24 hours of transfection, and the results are compared in Figure 4A. The comparison shows that none of the culture plates coated with gel, vacant $\mathrm{CS} / \mathrm{HA} \mathrm{NPs}$ or $\mathrm{CS} / \mathrm{HA} / \mathrm{miR}-21 \mathrm{NPs}$ exhibited any obvious cytotoxicity compared with the unmodified surface $(P>0.05)$.

To assess the ability of hBMMSCs to internalize the CS/ HA/miR-21 NPs, Cy3-labeled miRNAs and FITC-labeled CS were adopted to prepare fluorescently labeled CS/HA/miR-21 NPs, which were applied in the coating process.

Flow cytometry was employed to evaluate the transfection efficacy after transfection for 24 hours and 14 days (Figure 4B). The results indicated that different loading concentrations of miR-21 induced different transfection efficacies. In total, the transfection efficacy increased in

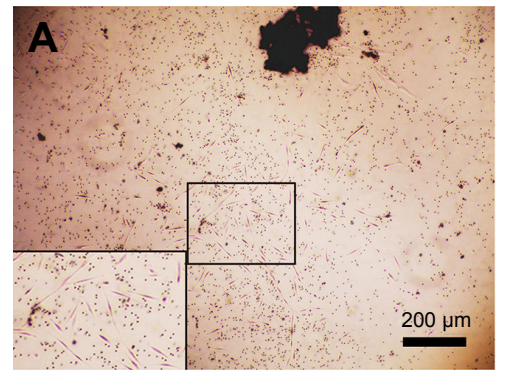

D STRO-1

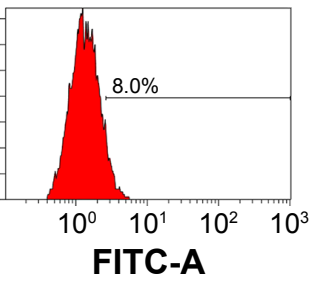

CD146
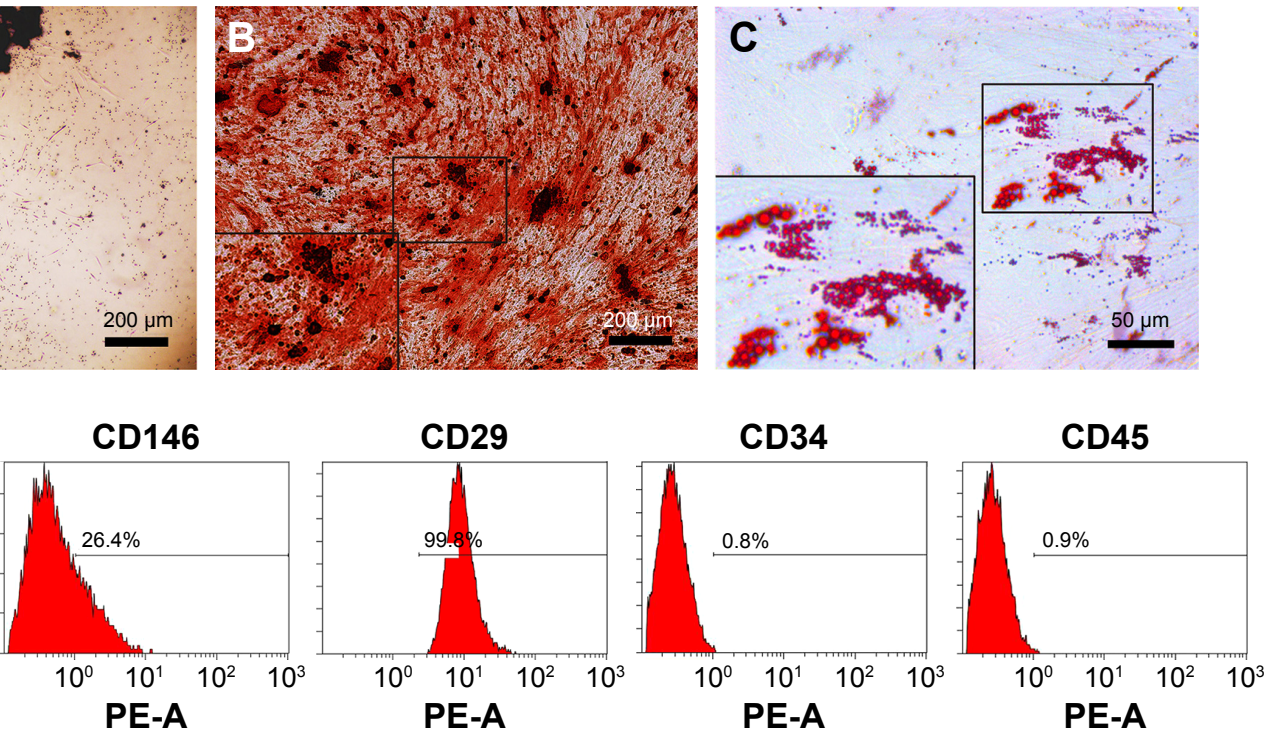

Isotype ctrl

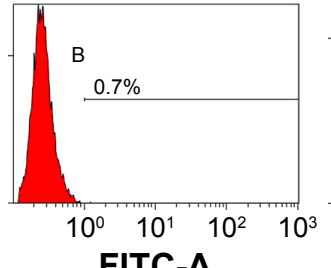

FITC-A
Isotype ctrI

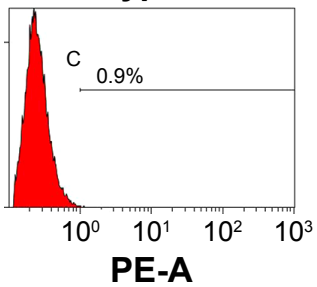

Figure 3 The isolation and characterization of hBMMSCs.

Notes: (A) The spindle-shaped stem cells grown in $\alpha$-MEM complete medium (100X). (B) The formed mineralized nodules were stained positively with alizarin red $\mathrm{S}$ after 4 weeks of osteogenic induction $(100 \times)$. (C) The microscopic oil red O-positive lipid droplets were observed after 2 weeks of adipogenic induction (400x). (D) Cytometric flow analysis showed that the presumed stem cells expressed the positive mesenchymal-associated markers STRO-I, CD29, and CDI46, but the cells were negative for the hematopoietic markers CD34 and CD45.

Abbreviations: hBMMSCs, human bone marrow mesenchymal stem cells; $\alpha$-MEM, $\alpha$-minimum essential medium; FITC, fluorescein isothiocyanate; Ctrl, control; PE, phycoerythrin. 

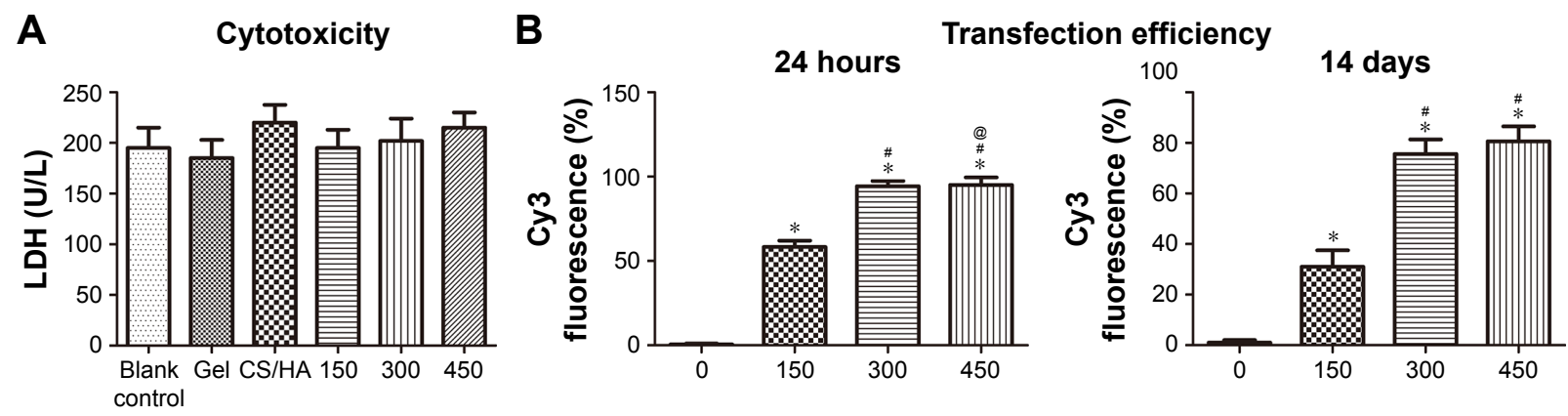

Figure 4 Cytotoxicity and transfection efficiency of the CS/HA/miR-2I NP-coated surfaces.

Notes: (A) The LDH amount released by cells during the first 24 hours after transfection. Blank control: unmodified surface; Gel: $0.2 \%$ gel solution-coated surface; CS/HA: the vacant CS/HA NP-coated surface; I50-450: CS/HA/miR-2I NP-coated surface at the concentration of $150-450$ pmol/well miR-2I. (B) Comparison of the transfection efficiency on different surfaces by flow cytometry. ${ }^{*} P<0.05$ versus the unmodified surface; $P<0.05$ versus the I50 pmol/well miR-2I group; @ $P<0.05$ versus the $300 \mathrm{pmol} /$ well miR-2I group.

Abbreviations: CS, chitosan; HA, hyaluronic acid; miR, microRNA; NP, nanoparticle; LDH, lactate dehydrogenase; h, hours.

a dose-dependent manner, and the $300 \mathrm{pmol} /$ well concentration yielded comparable effects to those of the $450 \mathrm{pmol} / \mathrm{well}$ concentration over the observation time; hence, we adopted the $300 \mathrm{pmol} / \mathrm{well}$ concentration in the subsequent coating process for cell sheet transfection.

After transfection for 1 day, 3 days, 5 days, 7 days, 10 days, and 14 days, fluorescent microscopy was used to track the internalized NPs during the process of hBMMSC sheet incubation (Figure 5). The results clearly showed that FITC-labeled CS and Cy3-labeled miR-21 accumulated within the bodies of cells, especially near the nuclear regions. The merged pictures demonstrated a high degree of overlap between FITC-labeled CS and Cy3-labeled miR-21, in turn indicating stable settlement within CS/HA NPs for miR-21 at each time point. Both the fluorescence intensities of FITClabeled CS and Cy3-labeled miR-21 decreased gradually in a time-dependent manner, but they could still be observed by fluorescence microscope at the end time point of 14 days, indicating a moderate and long-lasting transfection process during hBMMSC sheet induction. To confirm these results, we also employed Western blot to validate the efficacy of the delivery of miR-21, SPRY1 was reported to be direct target of miR-21. The Western blot results revealed that SPRY1 was repressed by miR-21 during the 14 days of cell sheet incubation (Figure 6A).

\section{Osteogenic gene expression}

After 14 days of cell sheet induction, the hBMMSC sheets were further incubated with the osteoinductive medium for 3 days, 7 days, and 14 days. At each time point, osteogenic gene expression, including $C O L 1, R U N X 2, O P N$, and $O C N$, on different surfaces was measured by real-time PCR (Figure 6B). The results indicated that different surfaces induced different gene expression levels in a time-dependent manner. Generally, the miR-21-functionalized surfaces demonstrated higher gene expression to varying degrees than unmodified surfaces and vacant CS/HA NP-coated surfaces. The miR-21-functionalized surfaces induced the highest COL1 1 and RUNX2 gene expression on day $3(P<0.05)$, while there were no obvious differences between unmodified surfaces and vacant CS/HA NP-coated surfaces for these two gene expressions. During the sheet incubation process of 14 days, the miR-21-functionalized surfaces yielded the highest expression of $O P N$ on day $7(P<0.05)$, while they showed the highest expression of $O C N$ on day $14(P<0.05)$ among the three groups. Further, the vacant CS/HA NPcoated surfaces tended to induce relatively higher expression of $O P N$ and $O C N$ compared to unmodified surfaces. Furthermore, comparing the relative protein expression profiles of cell sheets induced on different surfaces via Western blot (Figure 6C), the results showed that the miR-21-delivered hBMMSC sheets exhibited higher expression of calcificationrelated proteins (COL1, RUNX2, OPN, and OCN) to various degrees compared to cell sheets cultured on unmodified surfaces and vacant $\mathrm{CS} / \mathrm{HA} \mathrm{NP}$-coated surfaces.

\section{In vitro osteogenic differentiation of hBMMSC sheets}

Cell sheets were cultured on miR-21-functionalized surfaces of culture plates or other surfaces for 14 days and then incubated in osteogenic differentiation medium for 7 days and 14 days. On day 7 of osteogenic differentiation, the ALP activities of cell sheets were detected by staining and are expressed as the mean IOD of the images assessed using Image-Pro Plus 6.0 software (Figure 7A). The results showed that, in the miR-21-delivered cell sheets, the ALP production was significantly increased compared to the two controls $(P<0.05)$. After 7 days of osteogenic induction, the delivered 

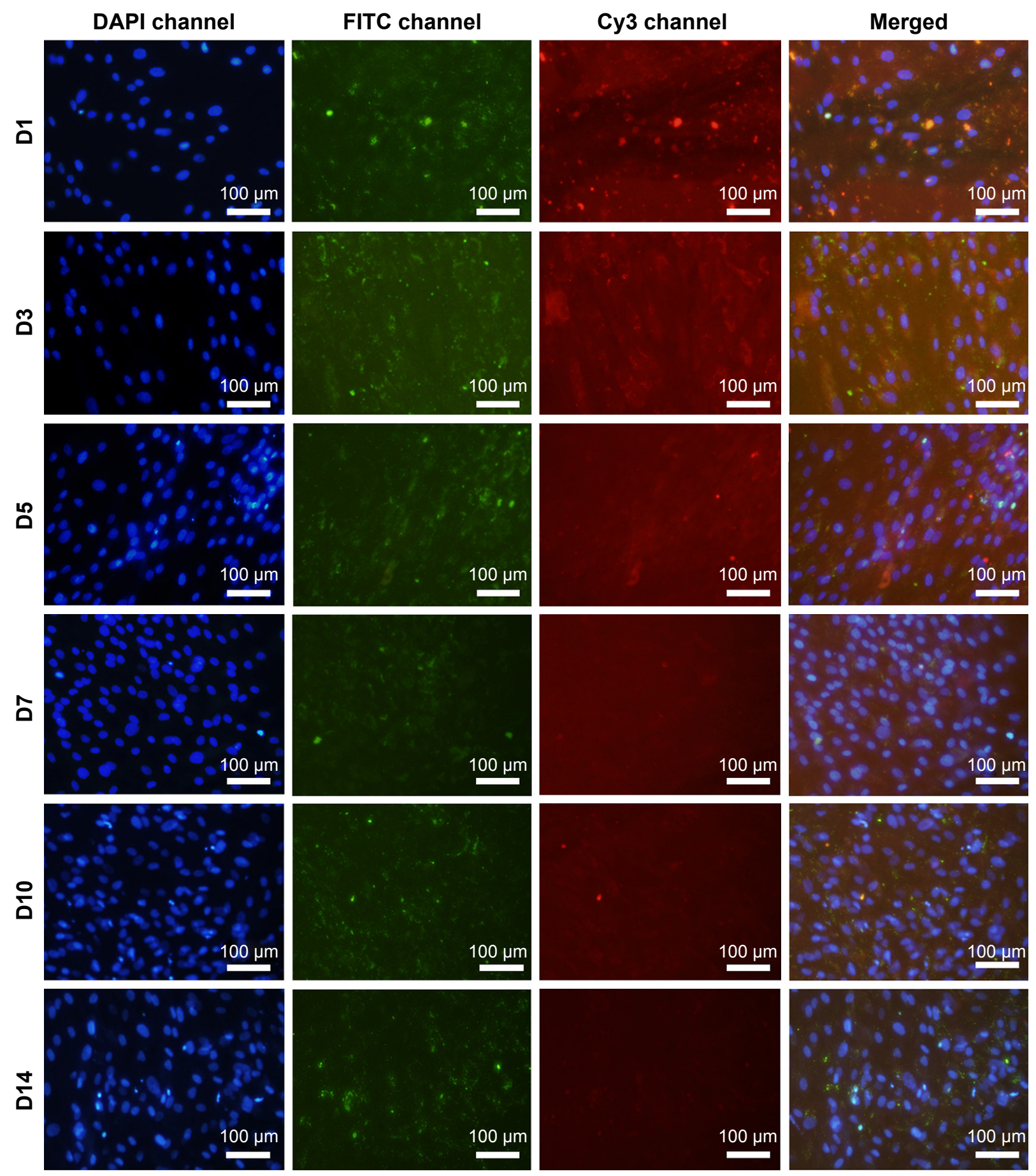

Figure 5 The track of fluorescently labeled CS/HA/miR-2I NPs after transfection for I day, 3 days, 5 days, 7 days, 10 days, and I4 days.

Notes: The pictures illustrate fluorescein DAPI-labeled nuclei (blue), FITC-labeled CS (green), Cy3-labeled miR-2Is (red) and merged images. The concentration of $300 \mathrm{pmol} /$ well miR-2I was adopted here for reverse transfection. Magnification: 200x.

Abbreviations: CS, chitosan; D, day; HA, hyaluronic acid; miR, microRNA; NP, nanoparticle; DAPI, 4',6-diamidino-2-phenylindole; FITC, fluorescein isothiocyanate.

cell sheets showed slightly deeper Sirius red staining compared to the controls, and the comparative trend in collagen secretion among the different groups was similar to that of ALP (Figure 7B). On day 14 of osteogenic differentiation, all the cell sheets exhibited the potential to undergo osteogenic differentiation based on positive Alizarin Red S staining, while the miR-21-delivered cell sheets appeared to accumulate the largest amount of calcium deposits, with more and larger mineralized nodules. After semi-quantification of ECM-mineralized nodules, miR-21-delivered cell sheets displayed much higher osteogenic potential than the other cell sheets $(P<0.05$; Figure $7 \mathrm{C})$; the ECM mineralization by 


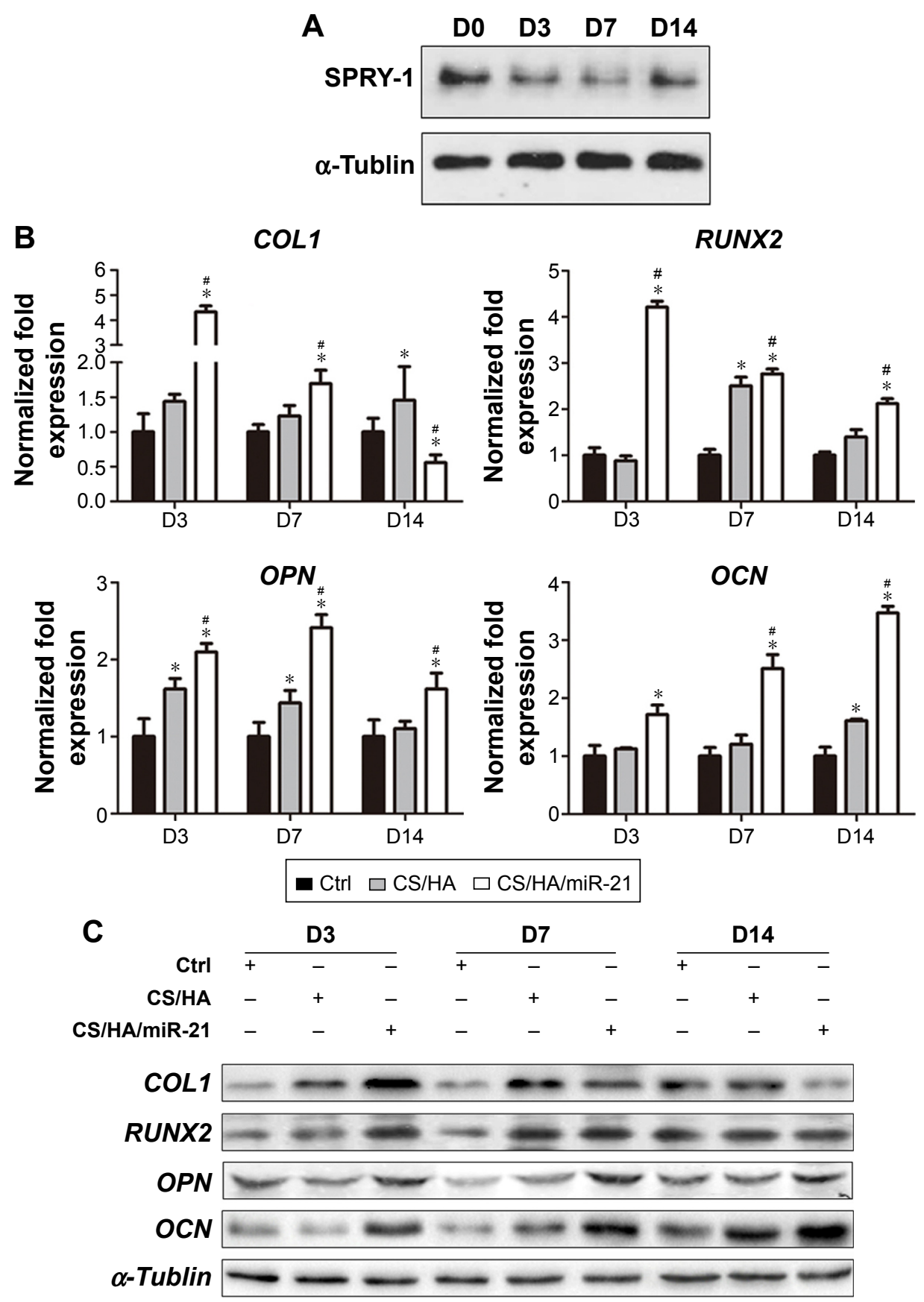

Figure 6 The expressions of related genes and proteins on the CS/HA/miR-2I NP-coated surfaces.

Notes: (A) SPRYI was repressed by miR-2I during the 14 days of cell sheet incubation. (B and C) Cell sheets were cultured on miR-2I-functionalized surfaces of culture plates or other surfaces for 14 days and then incubated in osteogenic differentiation medium for 3 days, 7 days, and 14 days. All values of real-time PCR were normalized to GUSB. $* P<0.05$ versus the unmodified surface (ctrl); $\# P<0.05$ versus the vacant $C S / H A$ NP-coated surface.

Abbreviations: CS, chitosan; D, day; HA, hyaluronic acid; miR, microRNA; NP, nanoparticle; PCR, polymerase chain reaction; GUSB, glucuronidase, beta; COLI, collagen type $|\alpha|$; RUNX2, runt-related transcription factor 2; OPN, osteopontin; OCN, osteocalcin.

the miR-21-delivered cell sheets was approximately 1.8 - to 2.5 -fold that of the controls.

\section{Discussion}

In recent years, stem-cell therapy for tissue defect repair/ regeneration has been advocated, and cell sheet engineering has emerged as a preferable delivery model for seeding cells due to well-preserved intact cell-cell junctions and cellular microenvironments with excellent biological and mechanical properties. ${ }^{23}$ Thus, it would be highly significant to explore further the performance by DNA or RNA delivery. ${ }^{8}$ In this work, we aimed to verify the feasibility of miR-21 delivery to cell sheets, with a safer nonviral approach than the viral approach, for enhanced osteogenic potential. In this study, we 


\section{A Noncoated}
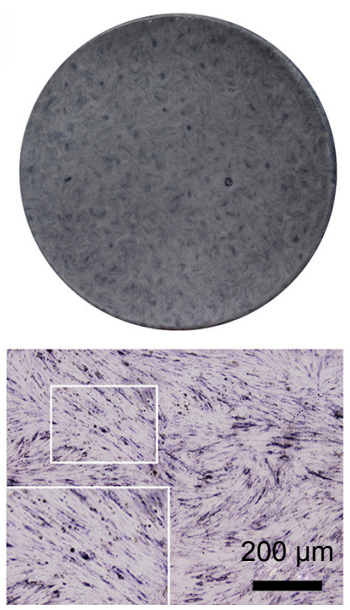

B
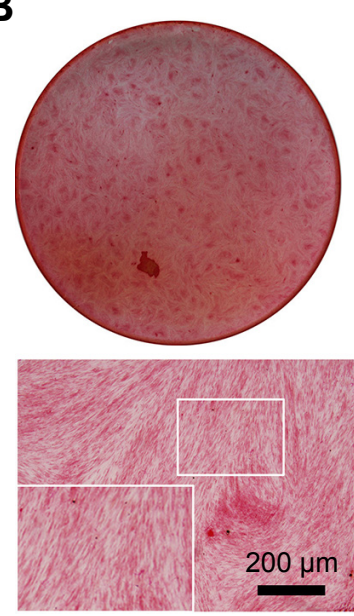

C
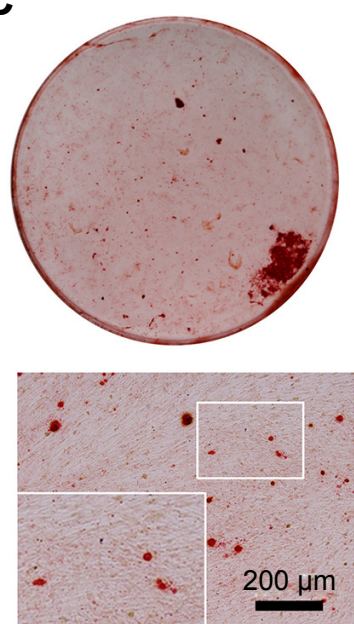
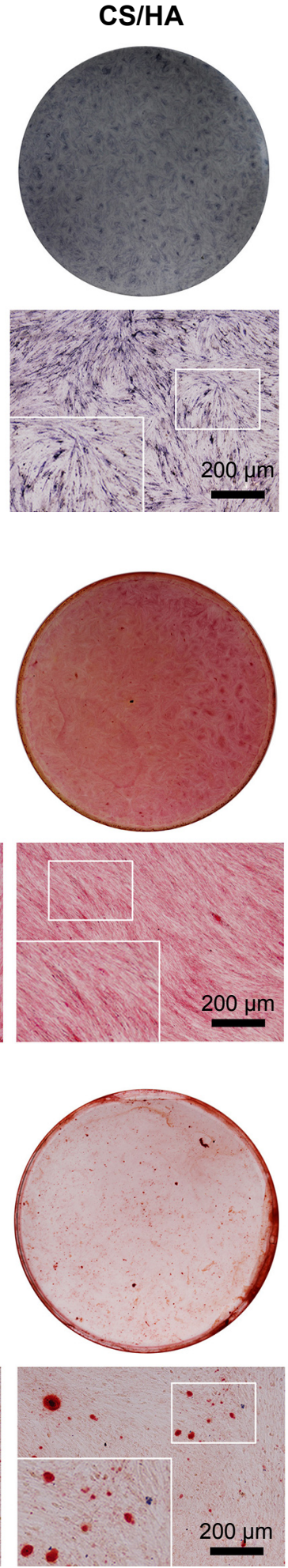

CS/HA/miR-21
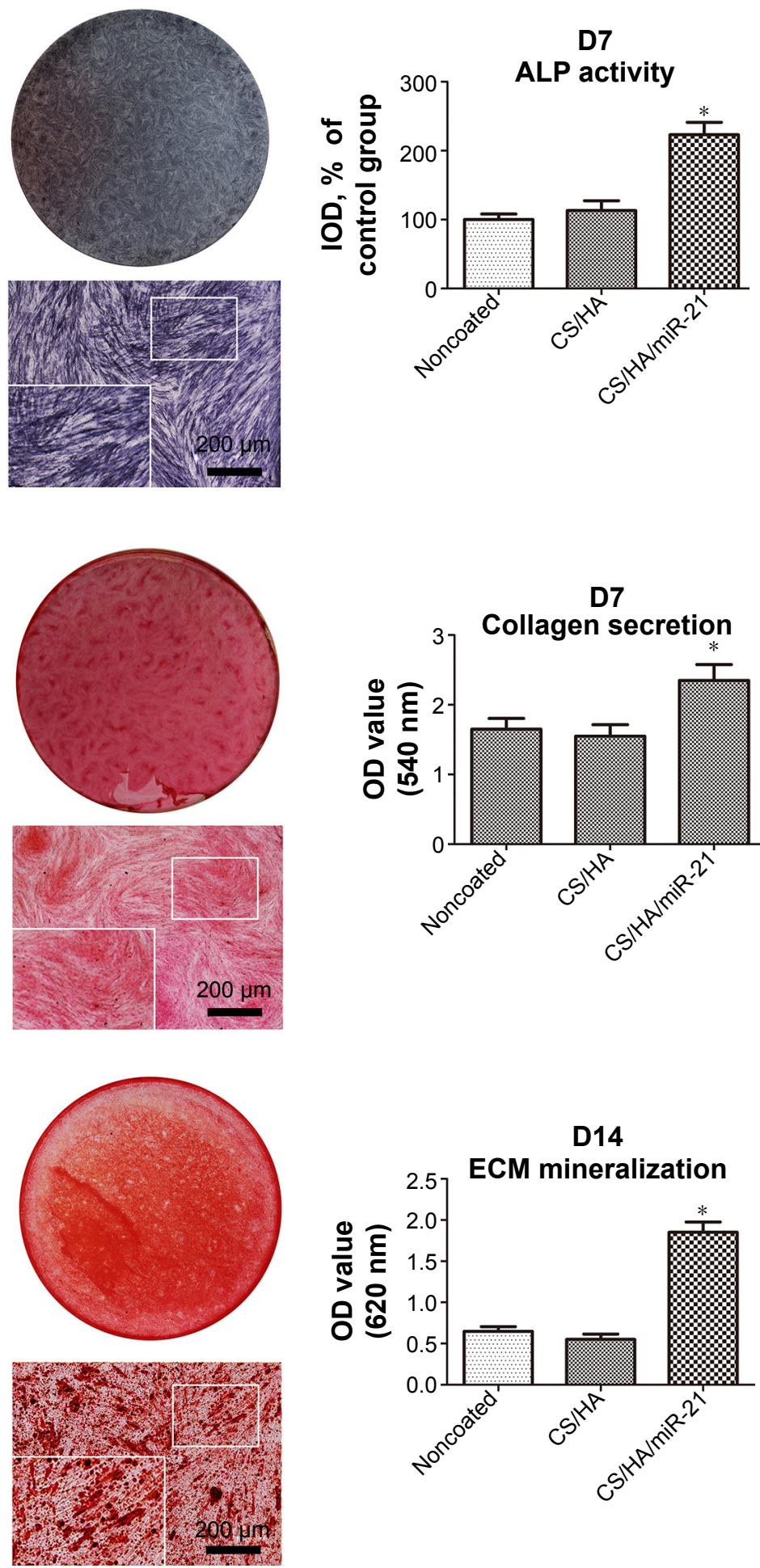

D7

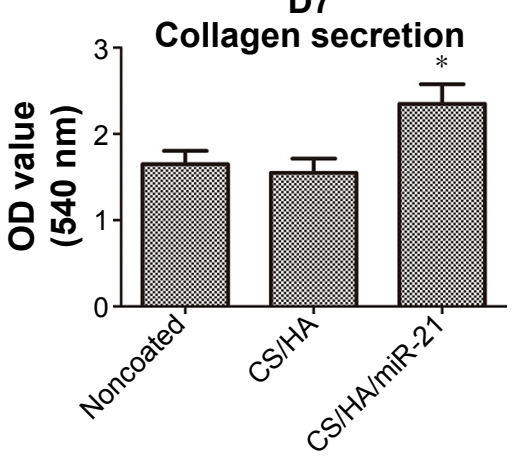

D14

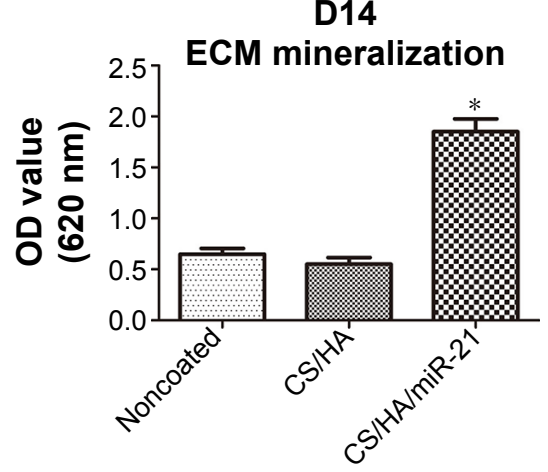

Figure 7 The characterization of osteogenic differentiation of hBMMSC sheets in vitro.

Notes: (A) Representative images and semi-quantitative analysis for ALP staining of the hBMMSC sheets after a 7-day osteogenic induction in vitro. $* P<0.05$ versus the unmodified surface and the vacant CS/HA NP-coated surface. (B) Representative images and semi-quantitative analysis for collagen secretion of the hBMMSC sheets after a 7-day osteogenic induction in vitro. $* P<0.05$ versus the unmodified surface and the vacant CS/HA NP-coated surface. (C) Representative images and semi-quantitative analysis for ECM mineralization of the hBMMSC sheets after a I4-day osteogenic induction in vitro. $* P<0.05$ versus the unmodified surface and the vacant CS/HA NP-coated surface. Magnification: I00x.

Abbreviations: hBMMSC, human bone marrow mesenchymal stem cell; ALP, alkaline phosphatase; CS, chitosan; D, day; HA, hyaluronic acid; NP, nanoparticle; ECM, extracellular matrix; miR, microRNA; OD, optical density; IOD, integrated optical density. 
developed a novel miR-21 reverse transfection formulation by cross-linking $\mathrm{CS} / \mathrm{HA} / \mathrm{miR}-21 \mathrm{NPs}$ on to tissue culture plates using $0.2 \%$ gel solution, after which the isolated hBMMSCs were seeded on to miR-21-functionalized culture plates and further incubated in cell sheet-inducing medium for 14 days. The assay results demonstrated that the hBMMSCs sheet could be successfully induced via the novel reverse transfection approach, and the miR-21 delivery significantly enhanced the in vitro osteogenic differentiation of hBMMSC sheets.

Generally, specially designed culture plates, such as temperature-responsive plates, are needed for cell sheet engineering, ${ }^{5}$ while, recently, a much simpler and more practical approach was developed by simply adding dexamethasone and $\mathrm{Vc}$ or $\mathrm{Vc}$ alone to the culture medium without requiring any specific equipment. ${ }^{6,8,23} \mathrm{Vc}$ was demonstrated to be capable of stimulating the ECM deposition of stem cells, thereby promoting cell sheet formation. ${ }^{6}$ In this work, the Vc-based method was chosen to generate the hBMMSC sheets. After 14 days of culture in the cell sheet-inducing medium, uniform cell sheets $\sim 50 \mu \mathrm{m}$ thick, containing two to five layers of cells, could be easily harvested from all the groups. In the miR-21-delivery group, the hBMMSC sheets were observed to maintain their structure well without discernible differences compared to the normally induced hBMMSC sheets. Using the cell sheet technique, the preserved ECM and mechanical properties could increase the cell survival rate and reduce cell loss during transplantation. In addition, we could conveniently manipulate the transplantation processes of stem cells, that is, we could control the size, amount, and location of transplanted cell sheets easily, or we could combine them with absorbable biomaterials for improved clinical effects. ${ }^{23,24}$

To improve further the therapeutic efficiency of cell sheets, we fabricated miRNA-delivery cell sheets. As one of the most studied miRNAs, miR-21 plays a general role in regulating a variety of physiological and pathological processes. ${ }^{25,26}$ Studies of embryonic stem cells and mesenchymal stem cells (MSCs) have shown that the levels of miR-21 increased substantially following the induction of differentiation, indicating that miR-21 might play important roles in stem cell differentiation and development. ${ }^{27}$ Among these roles, miR-21 was found to suppress stemness maintenance by downregulating SOX2 expression and promoting the osteogenic differentiation of hMSCs. ${ }^{25}$ To find a safe and efficient vector for gene delivery, we developed a $\mathrm{CS} / \mathrm{HA}$ nanocarrier system as a nonviral vector for reverse transfection. ${ }^{15} \mathrm{CS}$ possesses good biocompatibility and biodegradability, but a single application of CS lacks stability due to its low water solubility at physiological $\mathrm{pH}$, so CS likely could not protect miRNA efficiently, leading to the release of a large amount of miRNA before being endocytosed by the cells. ${ }^{22}$ However, the inclusion of an anionic polymer (HA) could increase the stability of the particles and increase the gene transfection efficacy, according to previous reports. ${ }^{16,20}$ In addition, HA could bind to CD44 on the hBMMSC cellular receptors, which would induce endocytosis in a targeted manner. ${ }^{21}$ Liu et $\mathrm{al}^{28}$ found that HA could improve cellular uptake through its binding receptor, leading to easy release of the transfection agents after internalization. ${ }^{20}$ In this work, an optimal mixture with a CS:TPP:HA volume ratio of 1:0.15:0.1 was adopted to prepare CS/HA NPs because our previous study validated that the loaded and unloaded NPs with a 1:0.15:0.1 ratio had the smallest PDI (best homogeneity of size distribution) compared to the other ratios, and the relatively high average zeta potential with this ratio also contributed to close contact with the anionic membranes of cells. ${ }^{16}$ These results were also consistent with other previous studies. ${ }^{29}$ In the gel retardation assay, the migration of miR-21 on agarose gel was completely retarded when the $\mathrm{N} / \mathrm{P}$ ratio was 20, indicating the complete combination of miR-21 with CS/HA NPs. As such, the N/P ratio of 20:1 was adopted to prepare $\mathrm{CS} / \mathrm{HA} / \mathrm{miR}-21 \mathrm{NPs}$ for this study.

As elaborated in the results, we successfully developed appropriate $\mathrm{CS} / \mathrm{HA} / \mathrm{miR}-21 \mathrm{NPs}$ and then cross-linked them on to the culture tissue plates using $0.2 \%$ gel solution to prepare a miR-21-functionalized surface. The SEM images showed that the miR-21-functionalized surface presented uniform and sleek CS/HA/miR-21 NPs, which formed a monolayer and were embedded tightly in the gel proteins. The merged images of Cy3-labeled miR-21s and FITClabeled CS demonstrated a high degree of overlap, indicating that the miR-21s were stably retained within the CS/HA NPs after the coating was prepared on the culture plates. In addition, these NPs could provide sustained release of miR-21 over a 14-day cell sheet induction process and could reduce cytotoxicity. This coating method was relatively simple and could be easily followed for potential clinical use. This work also demonstrated a relatively high level of transfection efficacy of $>90 \%$ after 24 hours of transfection and $>75 \%$ on day 14. Fluorescence observation revealed that the $\mathrm{Cy} 3-$ labeled miR-21s accumulated within the cells for at least 14 days, although with a gradual decrease in fluorescence intensity. These results indicated high transfection efficiency, 
which could be ascribed to reverse transfection, ${ }^{30,31}$ also known as substrate-mediated transfection. As designed in our study, CS/HA/miR-21 NPs were immobilized on to the surfaces of culture plates; therefore, the attached stem cells could directly contact these NPs, which would facilitate the endocytotic process of the cells and reduce the amount of floating transfection agents in the culture medium. ${ }^{32}$ The results were in agreement with previous reports of reverse transfection with small interfering RNA and miRNA. ${ }^{33}$

In this study, the assay results demonstrated that miR-21 delivery significantly enhanced the in vitro osteogenic differentiation of hBMMSC sheets, supported by upregulated calcification-related gene expression and enhanced ALP activity, collagen secretion, and mineralized nodule formation. The upregulated gene expression of COL1, RUNX2, $O P N$, and $O C N$ over time for the miR-21-delivered hBMMSC sheets indicated a rapid osteogenic induction process. Moreover, we found similar results at the protein expression level based on Western blot assay. RUNX2, COL1, and ALP activities are considered early indicators of osteoblast differentiation, while OPN is a mid-term indicator and OCN is a late indicator. The higher expression of these markers in miR-21-delivered cell sheets indicated rapid access to osteogenic differentiation. After semi-quantification of ECM mineralization, the miR-21-delivered cell sheets displayed much higher osteogenic capacity than the other cell sheets, which significantly enhanced the osteogenic differentiation of the hBMMSC sheets. Our results were in agreement with the following reports. Trohatou et $\mathrm{a}^{25}$ revealed that overexpression of miR-21 could accelerate osteogenesis and impair adipogenesis in hBMMSCs, and Yang et a ${ }^{34}$ validated that miR-21 could promote the osteoblast differentiation of hMMSCs by repressing SPRY1, which can negatively regulate the osteogenic differentiation of MSCs. Meng et al ${ }^{35}$ also found that miR-21 could promote the osteogenic differentiation of human umbilical cord mesenchymal stem cells through the PI3K-AKT-GSK3 $\beta$ pathway via the stabilization and accumulation of $\beta$-catenin. Our study confirmed the feasibility of fabricating miR-21-delivered hBMMSC sheets on CS/HA/miR-21 NP-coated culture plates by reverse transfection, and the results demonstrated higher osteogenic differentiation capability than that of the undelivered groups. The focus of our next study will be to determine the effects of these miR-21-delivered hBMMSC sheets in vivo as a novel stem-cell therapy in the field of bone tissue regeneration.

\section{Conclusion}

In this work, we aimed to verify the feasibility of miR-21 delivery to hBMMSC sheets for enhanced osteogenic potential.
First, we fabricated biocompatible and safe CS/HA NPs to deliver miR-21; then, we developed a novel miR-21 reverse transfection formulation by cross-linking CS/HA/miR-21 NPs on to tissue culture plates using $0.2 \%$ gel solution, after which the isolated hBMMSCs were seeded onto miR-21-functionalized culture plates and further incubated in cell sheet-inducing medium for 14 days. The assay results demonstrated that the hBMMSC sheets could be successfully induced via this novel reverse transfection approach, and the miR-21 delivery significantly enhanced the in vitro osteogenic differentiation of cell sheets in terms of upregulating calcification-related gene expression and enhancing ALP production, collagen secretion, and mineralized nodule formation. The enhanced osteogenic activity of cell sheets could promisingly lead to more rapid and robust bone regeneration for clinical use.

\section{Acknowledgments}

This study was financially supported by the National Natural Science Foundation of China (grant number 81200823) and the Shaanxi Province Science and Technology Research and Development Project (2014K11-01-02-13). The authors appreciate the Department of Toxicology, the Faculty of Preventive Medicine, the Fourth Military Medical University. $\mathrm{ZW}, \mathrm{GW}$, and MW are the co-first authors.

\section{Author contributions}

All authors contributed toward data analysis, drafting and critically revising the paper and agree to be accountable for all aspects of the work.

\section{Disclosure}

The authors report no conflicts of interest in this work.

\section{References}

1. Iwata $\mathrm{T}$, Washio $\mathrm{K}$, Yoshida $\mathrm{T}$, et al. Cell sheet engineering and its application for periodontal regeneration. J Tissue Eng Regen Med. 2015; 9(4):343-356.

2. Garbern JC, Lee RT. Cardiac stem cell therapy and the promise of heart regeneration. Cell Stem Cell. 2013;12(6):689-698.

3. Ding G, Liu Y, Wang W, et al. Allogeneic periodontal ligament stem cell therapy for periodontitis in swine. Stem Cells. 2010;28(10):1829-1838.

4. Akizuki T, Oda S, Komaki M, et al. Application of periodontal ligament cell sheet for periodontal regeneration: a pilot study in beagle dogs. J Periodontal Res. 2005;40(3):232-238.

5. Yamato M, Akiyama Y, Kobayashi J, Yang J, Kikuchi A, Okano T. Temperature-responsive cell culture surfaces for regenerative medicine with cell sheet engineering. Prog Polym Sci. 2007;32(8):1123-1133.

6. Wei F, Qu C, Song T, et al. Vitamin C treatment promotes mesenchymal stem cell sheet formation and tissue regeneration by elevating telomerase activity. J Cell Physiol. 2012;227(9):3216-3224.

7. Matsuura K, Utoh R, Nagase K, Okano T. Cell sheet approach for tissue engineering and regenerative medicine. J Control Release. 2014;190: 228-239.

8. Yan J, Zhang C, Zhao Y, et al. Non-viral oligonucleotide antimiR-138 delivery to mesenchymal stem cell sheets and the effect on osteogenesis. Biomaterials. 2014;35(27):7734-7749. 
9. Ohki T, Yamato M, Ota M, et al. Prevention of esophageal stricture after endoscopic submucosal dissection using tissue-engineered cell sheets. Gastroenterology. 2012;143(3):582-588.e1-e2.

10. Nishida K, Yamato M, Hayashida Y, et al. Corneal reconstruction with tissue-engineered cell sheets composed of autologous oral mucosal epithelium. N Engl J Med. 2004;351(12):1187-1196.

11. Carthew RW, Sontheimer EJ. Origins and mechanisms of miRNAs and siRNAs. Cell. 2009;136(4):642-655.

12. Cohen SG, Dzury DS, Michelini FJ. Staphylococcus aureus antigens in hypersensitivity reactions and experimental arterial sensitization. J Allergy. 1957;28(6):531-539.

13. Bushati N, Cohen SM. microRNA functions. Annu Rev Cell Dev Biol. 2007;23:175-205.

14. El-Aneed A. An overview of current delivery systems in cancer gene therapy. J Control Release. 2004;94(1):1-14.

15. Wang Z, Wu G, Feng Z, et al. Microarc-oxidized titanium surfaces functionalized with microRNA-21-loaded chitosan/hyaluronic acid nanoparticles promote the osteogenic differentiation of human bone marrow mesenchymal stem cells. Int J Nanomedicine. 2015;10:6675-6687.

16. Wu G, Feng C, Hui G, et al. Improving the osteogenesis of rat mesenchymal stem cells by chitosan-based-microRNA nanoparticles. Carbohydr Polym. 2016;138:49-58.

17. Holmes CA, Tabrizian M. Substrate-mediated gene delivery from glycol-chitosan/hyaluronic acid polyelectrolyte multilayer films. ACS Appl Mater Interfaces. 2013;5(3):524-531.

18. Zheng F, Shi XW, Yang GF, et al. Chitosan nanoparticle as gene therapy vector via gastrointestinal mucosa administration: results of an in vitro and in vivo study. Life Sci. 2007;80(4):388-396.

19. Ito T, Iida-Tanaka N, Niidome T, et al. Hyaluronic acid and its derivative as a multi-functional gene expression enhancer: protection from non-specific interactions, adhesion to targeted cells, and transcriptional activation. J Control Release. 2006;112(3):382-388.

20. Duceppe N, Tabrizian M. Factors influencing the transfection efficiency of ultra low molecular weight chitosan/hyaluronic acid nanoparticles. Biomaterials. 2009;30(13):2625-2631.

21. Knudson CB, Knudson W. Hyaluronan-binding proteins in development, tissue homeostasis, and disease. FASEB J. 1993;7(13):1233-1241.

22. Lu HD, Zhao HQ, Wang K, Lv LL. Novel hyaluronic acid-chitosan nanoparticles as non-viral gene delivery vectors targeting osteoarthritis. Int J Pharm. 2011;420(2):358-365.
23. Zhao YH, Zhang M, Liu NX, et al. The combined use of cell sheet fragments of periodontal ligament stem cells and platelet-rich fibrin granules for avulsed tooth reimplantation. Biomaterials. 2013; 34(22):5506-5520.

24. Dominici M, Le Blanc K, Mueller I, et al. Minimal criteria for defining multipotent mesenchymal stromal cells. The International Society for Cellular Therapy position statement. Cytotherapy. 2006;8(4): 315-317.

25. Trohatou O, Zagoura D, Bitsika V, et al. Sox2 suppression by miR-21 governs human mesenchymal stem cell properties. Stem Cell Transl Med. 2014;3(1):54-68.

26. Pan X, Wang ZX, Wang R. MicroRNA-21: a novel therapeutic target in human cancer. Cancer Biol Ther. 2010;10(12):1224-1232.

27. Krichevsky AM, Gabriely G. miR-21: a small multi-faceted RNA. J Cell Mol Med. 2009;13(1):39-53.

28. Liu Y, Kong M, Cheng XJ, Wang QQ, Jiang LM, Chen XG. Selfassembled nanoparticles based on amphiphilic chitosan derivative and hyaluronic acid for gene delivery. Carbohydr Polym. 2013;94(1): 309-316.

29. Deng X, Cao M, Zhang J, et al. Hyaluronic acid-chitosan nanoparticles for co-delivery of MiR-34a and doxorubicin in therapy against triple negative breast cancer. Biomaterials. 2014;35(14):4333-4344.

30. Wang Z, Wu G, Bai S, et al. MAPs/bFGF-PLGA microsphere compositecoated titanium surfaces promote increased adhesion and proliferation of fibroblasts. Biomed Mater. 2014;9(3):035006.

31. Zhang YM, Bataillon-Linez P, Huang P, et al. Surface analyses of micro-arc oxidized and hydrothermally treated titanium and effect on osteoblast behavior. J Biomed Mater Res A. 2004;68(2):383-391.

32. Ziauddin J, Sabatini DM. Microarrays of cells expressing defined cDNAs. Nature. 2001;411(6833):107-110.

33. Re F, Zanetti A, Sironi M, et al. Inhibition of anchorage-dependent cell spreading triggers apoptosis in cultured human endothelial cells. JCell Biol. 1994;127(2):537-546.

34. Yang N, Wang G, Hu C, et al. Tumor necrosis factor $\alpha$ suppresses the mesenchymal stem cell osteogenesis promoter miR-21 in estrogen deficiency-induced osteoporosis. J Bone Miner Res. 2013;28(3): 559-573.

35. Meng YB, Li X, Li ZY, et al. microRNA-21 promotes osteogenic differentiation of mesenchymal stem cells by the PI3K/ $\beta$-catenin pathway. J Orthop Res. 2015;33(7):957-964.
International Journal of Nanomedicine

\section{Publish your work in this journal}

The International Journal of Nanomedicine is an international, peerreviewed journal focusing on the application of nanotechnology in diagnostics, therapeutics, and drug delivery systems throughout the biomedical field. This journal is indexed on PubMed Central, MedLine, CAS, SciSearch $®$, Current Contents $\AA /$ Clinical Medicine,

\section{Dovepress}

Journal Citation Reports/Science Edition, EMBase, Scopus and the Elsevier Bibliographic databases. The manuscript management system is completely online and includes a very quick and fair peer-review system, which is all easy to use. Visit http://www.dovepress.com/ testimonials.php to read real quotes from published authors. 